Ebisu Ebisu

Études japonaises Études japonaises

49 | printemps-été 2013

De chose en fait : la question du milieu

\title{
Michel WASSERMAn, Claudel Danse Japon, Paris
}

Classiques Garnier, coll. "Études de littérature des $\mathrm{XX}^{\mathrm{e}}$ et $\mathrm{XXI} \mathrm{I}^{\mathrm{e}}$ siècles », 23, 2011, $158 \mathrm{p}$.

\section{François Lachaud}

\section{OpenEdition}

\section{Journals}

Édition électronique

URL : http://journals.openedition.org/ebisu/837

DOI : 10.4000/ebisu.837

ISSN : 2189-1893

Éditeur

Institut français de recherche sur le Japon à la Maison franco-japonaise (UMIFRE 19 MEAE-CNRS)

\section{Édition imprimée}

Date de publication : 1 avril 2013

Pagination : 175-180

ISSN : 1340-3656

\section{Référence électronique}

François Lachaud, « Michel wasserman, Claudel Danse Japon, Paris », Ebisu [En ligne], 49 | printemps-été 2013, mis en ligne le 25 mars 2014, consulté le 22 septembre 2020. URL : http:// journals.openedition.org/ebisu/837 ; DOI : https://doi.org/10.4000/ebisu.837

Ce document a été généré automatiquement le 22 septembre 2020.

(c) Institut français de recherche sur le Japon à la Maison franco-japonaise 


\section{Michel WASSERMAN, Claudel Danse Japon, Paris}

Classiques Garnier, coll. "Études de littérature des $\mathrm{XX}^{\mathrm{e}}$ et $\mathrm{XXI} \mathrm{I}^{\mathrm{e}}$ siècles ", 23, 2011, $158 \mathrm{p}$.

François Lachaud

\section{RÉFÉRENCE}

Michel WASSERMAN, Claudel Danse Japon, Paris, Classiques Garnier, coll. « Études de littérature des $\mathrm{xx}^{\mathrm{e}}$ et $\mathrm{XxI}^{\mathrm{e}}$ siècles », 23, 2011, 158 p. dont 12 ill. paginées en cahier central (p. 61-72).

1 De livre en livre, Michel Wasserman compose une œuvre où se croisent l'étude de l'attrait irrésistible exercé par la culture japonaise sur la création artistique en Occident - notamment en France - et, parallèlement, l'exploration des divers héritages de l'art occidental (à commencer par la musique) au sein de la culture artistique du Japon moderne et contemporain - Le Sacre de l'hiver. La Neuvième Symphonie de Beethoven, un mythe de la modernité japonaise (Paris, Les Indes Savantes, 2006). Les «mythologiques musicales» de M. Wasserman sont les fruits succulents d'une longue méditation nourrie de sa connaissance intime du Japon, de ses formes d'art dramatique, sans oublier la passion de la musique (occidentale et japonaise), ainsi que de l'expérience de la mise en scène lyrique et des échanges culturels (direction de l'Institut franco-japonais du Kansai; et de la villa Kujoyama). Il ajoute avec Claudel Danse Japon l'éclairage - cette essence si volatile de tout spectacle réussi - d'une étude de cas composée avec virtuosité.

2 Dans son précédent ouvrage, D'Or et de Neige. Paul Claudel et le Japon (Paris, Gallimard, coll. «Les Cahiers de la NRF», 2008), M. Wasserman avait retracé l'impressionnant tableau des relations entre l'œuvre claudélien et le Japon; ce pays qui ne cessa d'agir pour l'illustre homme de lettres à la fois comme une haute terre du songe, un moment important de son action diplomatique et une véritable dynamo alimentant une 
nouvelle phase de création littéraire et dramatique intense: on s'en tiendra ici à la mention de L'Oiseau noir dans le Soleil levant de 1926, des Cent Phrases pour éventail de 1927 (publié au Japon en collaboration avec un peintre lettré de renom) et le Soulier de satin de 1929 ; "synthèse " de son art dans toute sa démesure. La passion pour le Japon pourrait sembler n'être qu'une anecdote dans l'œuvre proliférant de Claudel. Toutefois, malgré les invectives (qui ne se souvient des anathèmes des Surréalistes ?) et les années d'ostracisme parmi les bien-pensants des deux rives, Claudel demeure l'une des figures les plus marquantes de la «modernité dramatique » qu'aucun spécialiste du Japon ne peut ignorer sinon à ses risques et périls. Avec Samuel Beckett (1906-1989), il reste l'un des auteurs dramatiques francophones les plus admirés du $\mathrm{xx}^{\mathrm{e}}$ siècle - la nouvelle édition dirigée par Michel Autrand de son Théâtre (Paris, Gallimard, coll. «Bibliothèque de la Pléiade », 2011, 2 vols.) fournit, sur près de 4000 pages, l'une des plus irréfutables preuves de cette vitalité. Paul Claudel, grâce aux liens singuliers qu'il cultiva avec les artistes japonais, incarne une forme de création artistique dans laquelle l'étranger ne se cantonne plus à l'exotisme du figurant ou à une altérité à laquelle on se heurte, mais devient un véritable ressort de la création.

Claudel Danse Japon étudie les métamorphoses, en un sens pleinement ovidien, d'un ballet conçu au Brésil en collaboration avec Darius Milhaud (1892-1974) à l'intention de Vaslav Nijinski (1889-1950) intitulé L'Homme et son désir (1917-1918), devenu " mimodrame" pour le kabuki sous le titre de la Femme et son ombre (1922-1923). On le sait, Nijinski sombra dans la maladie mentale avant que de pouvoir jouer le ballet brésilien (une ancienne malédiction lui aurait prédit ce sort funeste dût-il traverser un jour l'Atlantique). Son double, Nakamura Fukusuke V 中村福助 (1900-1933), acteur légendaire de kabuki d'une beauté physique hors du commun qui lui permettait d'atteindre à une quasi perfection dans les rôles féminins - les seuls qu'il jouait ( $\mathrm{j}$. maonnagata 眞女形) à la différence d'acteurs plus versatiles - devait mourir dans la fleur de l'âge. Un semblable destin tragique attendait le « remplaçant » de Nijinski, Jean Börlin (1893-1930). Parmi bien d'autres acteurs aujourd'hui négligés, le livre rend justice à ce chorégraphe et danseur suédois qui fit l'admiration du Tout-Paris dans des œuvres aussi diverses que Skating Rink, issu d'une collaboration avec Arthur Honegger (1892-1955) et Fernand Léger (1881-1955), ou dans la « danse du fétiche » de Scuplture nègre (1920) sur une musique de Francis Poulenc (1899-1963) avec des décors et costumes de Paul Colin (1892-1985).

Sur le modèle des sonates mozartiennes, le premier mouvement de l'ouvrage est consacré à la gestation brésilienne de L'Homme et son désir («Rio», p. 15-45), le deuxième à un intermède danois, puis à la scène parisienne et à la première ou « avantpremière générale » (« Paris », p. 47-82), enfin le dernier mouvement, appelé « Tokyo » (p. 83-122) est consacrée à l'avatar japonais du ballet initial. Les pages bruissent d'anecdotes et de portraits, de sources encore inexplorées qui se fondent avec harmonie à l'ensemble d'une richesse surprenante.

5 Mal gré qu'on en ait, Paul Claudel ne se situe jamais là où on l'attend, ne se conforme pas à sa légende noire et participe sans ciller, aux côtés des avant-gardes de son temps, à un renouveau artistique qui perpétue, sans doute pour la dernière fois, le mythe fécond de Paris comme capitale artistique du monde. La genèse de son ballet tropical s'opère sur fond d'échanges franco-brésiliens en pleine guerre mondiale, tandis que les cours du café constituent le principal souci professionnel du poète-diplomate devenu épicier/grossiste à ses heures. On découvre un épisode « célinien » mentionné pour la 
première fois dans un article de Paris-Soir en 1936 sous le titre étonnant de : "Comment, au Brésil, nous fûmes ensevelis, moi, mon ambassade et un dictateur positiviste dans une explosion de siphons et d'apéritifs ». L'action diplomatique française au Brésil durant la période connue sous le nom de República Velha (1889-1930) forme un contrepoint étonnant au "poème plastique » de Claudel et de Milhaud. Le pays gigantesque, durant la dernière décade du régime de la Vieille République est alors dirigé par des oligarques admirateurs d'Auguste Comte - le coronelismo - et des fazendeiros (grands propriétaires) sans scrupules qui ressemblent à s'y méprendre à leur ancêtre fantasmatique dans la Vie parisienne (1866). Ces quarante années s'achevèrent avec la dictature de Getúlio Vargas (1883-1954); régime totalitaire qui, sous le nom d'Estado Novo, prit place dans le tourbillon funeste qui devait entraîner au pas de l'oie le monde vers le pire dans les années trente.

Lors du séjour brésilien de Claudel, la France s'oppose à la présence allemande (il en va de même pour son séjour au Japon) et s'efforce d'établir une politique à son avantage et de complaire aux élites locales. La méthode de M. Wasserman consiste à ne jamais opposer les archives diplomatiques, les sources journalistiques et l'activité créatrice sans oublier la vie sentimentale riche du poète. On remarque la «clairvoyance » du ministère des Affaires étrangères qui n'hésite pas à affecter Darius Milhaud en qualité de " secrétaire et chargé du service de la propagande ». Le compositeur fut l'un des plus fidèles admirateurs de Claudel, alors que tout semblait les devoir opposer. M. Wasserman, dans l'analyse serrée de la partition (méconnue) de L'Homme et son désir (op. 48), confirme que Milhaud fut sans doute le musicien le plus innovant des Six. Cet homme qui devait enseigner au pianiste Dave Brubeck l'art de garder ses «oreilles ouvertes » et de voyager à contretemps, faute de Japon, se souvenait lui-même avec passion des nuits de Harlem en 1922. Lors de son séjour avec Claudel, entre deux incursions aux lisières de l'immense et inquiétante forêt - anticipation de celle que décrirait en 1930 José Maria Ferreira de Castro (1898-1974) dans A Selva (Forêt vierge, mis en français par Blaise Cendrars en 1938) - parmi les villégiatures à Petrópolis dans la Serra dos Órgãos, Milhaud se montre à l'écoute de toutes les musiques brésiliennes, des polyphonies et de la syncope. Agitateur d'idées à l'instar de son ami, n'hésitant pas à saluer les « orages bienfaisants du jazz » ni à défendre Gounod, Verdi ou la musique folklorique française, Milhaud est bel et bien le compagnon de route idéal de Claudel, tout comme il fut, d'une tout autre manière, l'inspirateur de Claude Lévi-Strauss qui devait lui dédier l'un de ses derniers livres dont le titre est aussi celui de l'une des œuvres les plus célèbres du musicien: Saudades du Brasil (1920). Quant aux douze danses de Milhaud, elles s'achèvent par un hommage à Claudel. Impossible de ne pas s'interroger sur la «coïncidence» qui fait du grand anthropologue l'autre figure dont les pas d'abord orientés vers le Brésil s'arrêtèrent ensuite au Japon, «la face cachée du monde ».

7 On connaît assez bien la passion que Claudel nourrissait à l'endroit du théâtre nō et celle qu'il vouait au théâtre de marionnettes (j. bunraku 文楽); deux formes théâtrales auxquelles il consacra des essais importants. En revanche, Claudel Danse Japon montre que les conceptions scéniques du dramaturge français entretiennent des affinités électives avec le kabuki (M. Wasserman a traduit, rappelons-le, l'une des pièces les plus célèbres du répertoire en 1981). Certes, les spécialistes (on évitera le mot " claudéliens ») tendent à tirer La Femme et son ombre en direction du nō, faisant leurs les clichés défavorables à l'endroit du kabuki où pourtant les spectres féminins forment la matière de nombreuses pièces célèbres (l'illustration de la p. 73 représentant la scène 
du Théâtre impérial évoque immédiatement les histoires étranges et le goût pour les épisodes terrifiants du kabuki bien plus que les âmes errantes du nō). Le kabuki devait par ailleurs jouer un rôle plus important que le nō dans l'art dramatique moderne par sa capacité à s'adapter aux nouvelles réalités, à la contestation sociale et transposer celles-ci sur la scène - ainsi chez Kawatake Mokuami 河竹黑阿弥 (1816-1893), le premier et sans doute le plus important auteur moderne de kabuki dont les drames de mauvais garçons et des bas-fonds se montraient « brechtiens » avant l'heure.

Ce goût de l'hybride, du métissage des formes et des vases communicants forme la matière principale du livre Claudel Danse Japon. Il n'est nulle part plus évident que dans l'itinéraire du poète de Petrópolis à Nikkō, en passant par le Danemark (autre poste diplomatique) et Paris, d'une œuvre née de ces fécondations à répétition. Des selves du Brésil aux Ballets suédois - concurrents et dissidents des célèbres Ballets russes qui furent l'une des plus belles contributions au modernisme et à l'hybridation des cultures depuis les faces khmères du Dieu Bleu à l'Orient de Schéhérazade - jusqu'au rejet en terre japonaise du mimodrame joué par des acteurs de kabuki, le livre n'est pas la recension du parcours d'un chef-d'œuvre unanime. Tant s'en faut: la pièce sous ses dehors tropicaux où sa forme japonaise fut à l'origine d'un flot de critiques qui meurtrirent à jamais la vanité de l'auteur.

9 Le livre présente pour la première fois les dessins d'Audrey Parr (1892-1940) conçus pour les costumes de L'Homme et son désir et légendés par Claudel lui-même. Ceux-ci, tout comme la mémoire critique de l'œuvre, sont conservés aujourd'hui dans l'extraordinaire écrin du Dansmuseet (Musée de la Danse) de Stockholm. Audrey Parr (née Audrey Manuella Enriqueta Bapst) avait elle-même un père d'origine alsacienne et une mère anglaise issue d'une famille dans laquelle se croisaient ancêtres polonais et brésiliens. À l'exemple de Milhaud, elle est tout sauf une figure de second rang, mais plutôt une autre "voyageuse excentrique ", familière de l'aristocratie londonienne et éprise de l'œuvre de Claudel. Le poète-ambassadeur - surnom que les Japonais avaient donné à Claudel - passe, le temps de deux premières (juin 1921 et mars 1923), de la « mâle beauté » imaginée pour le faune Nijinski aux spectres féminins du Japon, tout en nouant des relations avec les intellectuels et les artistes japonais curieux des mouvements esthétiques d'Occident, mais également - l'exemple est plus rare - avec des personnalités issues de l'art traditionnel. En filigrane se lisent aussi les gouffres et les orages de sa vie amoureuse.

10 M. Wasserman rend compte de l'importance du travail du compositeur/ interprète Kineya Sakichi IV 杵屋佐吉 (1884-1945), véritable trublion dans le monde parfois hermétique et raide de la musique traditionnelle; cette grande oubliée des autorités de jadis et d'aujourd'hui. Kineya transforma la tradition musicale locale et n'hésita pas à associer aux formes musicales japonaises celles venues de la musique occidentale - forçant ses pairs à une scission entre détracteurs et admirateurs (la cicatrice reste béante). Il fût célébré à Londres comme à Paris pour son génie audacieux qui sut introduire le public occidental au monde de la musique traditionnelle tout en rendant hommage à des artistes comme Maurice Ravel (1875-1937) dont l'œuvre procède elle aussi de la confluence des formes. Parmi les artistes avec qui Claudel travaille, on découvre Kaburaki Kiyokata 鏑木清方 (1878-1972); sans doute l'un des peintres japonais les plus importants du xxe siècle, ami d'Izumi Kyōka 泉鏡花 (1873-1939) - le Protée de la littérature et de l'art dramatique modernes. Kaburaki fut aussi une figure majeure des arts graphiques, un décorateur de génie ainsi qu'un 
essayiste unanimement admiré pour la beauté de son style. En lui confiant les décors et les costumes de La Femme et son ombre, Claudel faisait montre, une fois encore, de sa capacité étonnante à rallier les artistes les plus remarquables autour de lui. On espère qu'une grande rétrospective rende, enfin, justice à ce " peintre considérable » (p. 106) auprès du public occidental.

11 L'ensemble de ces vies parallèles transforment à leur tour le livre en un plaidoyer pour une « connaissance de l'Asie » qui n'abdiquerait pas face aux exigeants défis que pose l'analyse des divers métissages culturels. Trempé dans son encre lumineuse, Claudel Danse Japon se fonde à égalité sur ses deux piliers de toute recherche que sont la rigueur et l'enthousiasme, tout parvenant à mettre en relation des mondes si différents, si proches. La lecture attentive de ces pages ne manquera de stimuler les amateurs de Claudel, de musique, de ballet, de dramaturgie, d'art japonais ou brésilien ; invitation parfaite à d'autres partages inspirés.

\section{AUTEURS}

\section{FRANÇOIS LACHAUD}

École française d'Extrême-Orient 